\title{
Cesium-Induced Acquired QT Prolongation Causing Ventricular Tachycardia and Torsades De Pointes: Fatal Complication of Over-the-Counter Supplements
}

\author{
Mahida Hetavi $^{\mathrm{a}, \mathrm{c}}$, Dawn Calderon ${ }^{\mathrm{b}}$, Ashish Patel ${ }^{\mathrm{b}}$, Mohammad A. Hossain ${ }^{\mathrm{a}}$
}

\begin{abstract}
Prolongation of the QT interval can be from congenital or acquired cause. It can lead to polymorphic ventricular tachycardia known as torsades de pointes (TdP). Many drugs have been known to prolong QT belonging to various pharmacologic classes, but most of them share one common pharmacological effect by lengthening the cardiac repolarization mostly by blocking specific cardiac $\mathrm{K}^{+}$channels which leads to development of membrane oscillations (early after depolarizations) due to $\mathrm{Ca}^{2+} / \mathrm{Na}^{+}$re-entry. Propagation early after depolarization leads to development of TdP. Cesium is an inorganic compound which has been shown to cause TdP in animal experimental model, but only few cases of high-dose cesium-induced bradycardia leading to QT prolongation have been reported in humans. We present a rare case of a lady who was taking low dose of cesium $500 \mathrm{mg}$ daily leading to bradycardia and prolonged QT thus reflecting the variability in dose-dependent effects of acquired long QT substrates.
\end{abstract}

Keywords: Torsades de pointes; Cesium; QT prolongation

\section{Introduction}

Cesium is an inorganic compound, which is not approved by the Food and Drug Administration (FDA), and is available as over-the-counter (OTC) drug. Despite lack of supportive data on efficacy, and evidence of safety of the cesium, this drug is used by the patients for treatment of cancer [1], depression and other conditions. There is also not enough data on how cesium therapy works. Cesium is available in low to high doses as oral medication. It has been reported that low dose of cesium can

Manuscript submitted May 11, 2019, accepted June 3, 2019

aDepartment of Medicine, Hackensack Meridian Health, Jersey Shore University Medical Center, Neptune, NJ 07753, USA

bepartment of Cardiology, Hackensack Meridian Health, Jersey Shore University Medical Center, Neptune, NJ 07753, USA

${ }^{\mathrm{c} C}$ Corresponding Author: Mahida Hetavi, Department of Medicine, Hackensack Meridian Health, Jersey Shore University Medical Center, Neptune, NJ 07753, USA. Email: Hetavi.mahida@hackensackmeridian.org cause nausea, vomiting and diarrhea, while high dose as much as $6 \mathrm{~g}$ can cause life-threatening cardiac complications such as arrhythmias, prolonged QT interval, cardiac arrest, low potassium and central nervous system (CNS) complications like seizures and fainting $[2,3]$. We herein present a rare case of acquired severe QT interval prolongation with even low dose of oral OTC cesium intake.

\section{Case Report}

A 77-year-old woman with past medical history of stage IV colon cancer, hypertension and diabetes presented with a witnessed cardiac arrest followed by cardiopulmonary resuscitation and successful return of spontaneous circulation in the field. Review of system was negative for chest pain, palpitation, abdominal pain and dizziness. Patient reported that she has been non-compliant with her antihypertensive and antidiabetic medications; however she has been taking OTC drug cesium to cure her cancer. Patient has no previous history of cardiac arrhythmias and no family history of prolonged long QT syndrome or sudden cardiac death. On examination patient has a pulse of 42 beats per min, blood pressure of 102/56, and temperature of 98.8. Complete physical examination was normal except for sinus bradycardia on auscultation. Her electrocardiogram (EKG) showed sinus bradycardia with markedly prolonged QT interval of 662 - $735 \mathrm{~ms}$ (Figs. 1, 2). Laboratory data showed potassium of $3.5 \mathrm{meq} / \mathrm{L}$ and magnesium of 1.5 $\mathrm{mg} / \mathrm{dL}$ with negative serial troponins. She was initially treated with multiple lidocaine boluses and intravenous (IV) drip followed by isoproterenol. An urgent coronary artery angiography revealed clean coronary arteries. A transvenous pacemaker was placed which resulted in abrupt termination of the torsade. An echocardiogram showed normal biventricular function with no motion abnormality. On further query to evaluate the cause of her torsades, she admitted that she was taking cesium as a holistic treatment for colon cancer. Cesium was immediately withdrawn and patient was monitored with serial electrocardiogram. While corrected QT interval (QTc) continued to

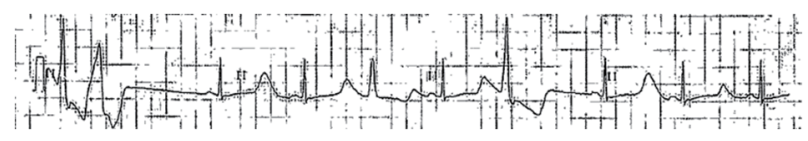

Figure 1. Sinus bradycardia with prolonged QT. 


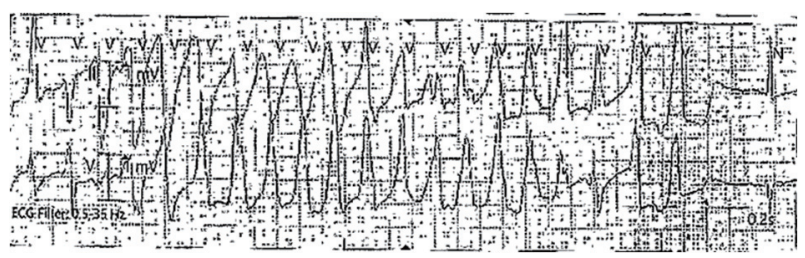

Figure 2. Patient having episode of polymorphic ventricular tachycardia.

improve patient developed paroxysmal atrial fibrillation (AF). A decision was made to implant a dual chamber pacemaker as she would need atrioventricular (AV) nodal blockers to treat her AF which could exacerbate the substrate for torsade. Dual chamber pacemaker was implanted. The patient had no further events and was discharged in the stable condition.

\section{Discussion}

Cesium is an alkali metal that has a radioactive isoform (cesium 137) and a non-radioactive salt (cesium chloride). Cesium 137 is used in radiation therapy. Cesium chloride, the non-radioactive salt, has been promoted by the "integrative medicine" practitioners as an alternative and natural treatment for various types of cancers [4]. We did a review of scientific literature on cesium chloride research. Although effects of this drug have been studied and reported in detail in animal models such as mice and dogs, there is no convincing human research study.

In our case, patient was taking cesium for stage IV colon cancer cure after intolerance and failed chemotherapy. It has been proposed that cesium chloride therapy also called "high $\mathrm{pH}$ therapy" reduces the acidity of tumor cells (raises their pH), which is described as very acidic. In 1984, Dr. Brewer et al reported in their publication entitled "The high $\mathrm{pH}$ therapy for cancer tests on mice and humans" that $\mathrm{pH}$ therapy has been shown to be successful in rats; however this study is not scientifically accepted $[5,6]$. It has also been proposed that cesium can almost treat any type of cancer including brain, heart, lung, breast, gastrointestinal and genitourinary cancers. Scientific evidence of clinical benefit from cesium in human cancer is limited to one case series published in 1984 by Sartori et al [1]. That case series has major flaws including its uncontrolled nature, retrospective design and probable case selection bias. Therefore, the results cannot be considered reliable. Another possible mechanism is that cesium neutralizes the toxic materials produced by cancer cells. This further prevents the mitosis of tumor cells and eventually leads to death of cell death. There is again no data to support this evidence as well [7].

Interestingly, most of the side effects of cesium toxicity are noticed with either high doses or long use of drug. In our case patient was taking small dose of $500 \mathrm{mg}$ of cesium. In a recent case, Lyon et al have reported that cesium toxicity was manifested by syncope, polymorphic ventricular tachycardia, hypokalemia, and a QT interval prolonged to $650 \mathrm{~ms}$ in patient who was taking $3 \mathrm{~g}$ /day [8]. Our patient developed prolonged QT interval and sudden cardiac arrest with low dose of cesium. It has been found that cesium can cause ventricular refractori- ness and prolonged QTc interval by affecting calcium channel in presence of electrolytes imbalance and bradycardia. Most drugs act by synergistic or antagonist effect. A slow driving rate and low potassium in the extracellular space produce a driving effect [9]. This case is evidence that even low doses can lead to this untoward effect.

It has also been observed that cardiac signs of cesium toxicity like prolonged QT interval are usually reversible, once the drug is stopped. However Chan et al [4] have reported another interesting scenario where cesium toxicity was managed by not only cessation of cesium exposure and vigorous electrolytes replacement but also oral Prussian blue therapy. Normalization of QT interval also varies patient to patient and level of toxicity.

Although FDA has either removed drugs causing QT prolongation or has listed them in "black box warning labels" [1], the potential effect of many dietary supplements such as cesium chloride remains unreported. Moreover these supplements can be easily purchased online or over the counter. Therefore, it is utmost important to consider these drugs among the differential diagnosis of prolonged QT interval [7].

\section{Conclusions}

In conclusion, even small dose of cesium could lead to fatal cardiac complications such as prolonged QT events and TdP leading to sudden cardiac arrest. A detail history with possible questionnaires about various non-prescribed, OTC supplement could give a clue about causative agent. Also, physician should counsel the patients on the life-threatening side effects of these medications.

\section{Acknowledgments}

None.

\section{Financial Disclosure}

This project was not supported by any grant or funding agency.

\section{Conflict of Interest}

The authors declare that there is no conflict of interest regarding the publication of this paper.

\section{Informed Consent}

The patient described in the case report had given informed consent for the case report to be published.

\section{Author Contributions}

MH contributed to writing of entire manuscript; DC and AP 
participated in case presentation; MAH participated in discussion writing.

\section{References}

1. Sartori HE. Cesium therapy in cancer patients. Pharmacol Biochem Behav. 1984;21(Suppl 1):11-13.

2. Aschenbrenner DS. Cesium chloride, used as alternative cancer therapy, poses risks. Am J Nurs. 2018;118(11):20.

3. Wiens M, Gordon W, Baulcomb D, Mattman A, Mock T, Brown R. Cesium chloride-induced torsades de pointes. Can J Cardiol. 2009;25(9):e329-331.

4. Chan CK, Chan MH, Tse ML, Chan IH, Cheung RC, Lam CW, Lau FL. Life-threatening Torsades de Pointes resulting from "natural" cancer treatment. Clin Toxicol (Phila). 2009;47(6):592-594.

5. Brewer AK. The high $\mathrm{pH}$ therapy for cancer tests on mice and humans. Pharmacol Biochem Behav. 1984;21(Suppl $1): 1-5$.

6. Low JC, Wasan KM, Fazli L, Eberding A, Adomat H, Guns ES. Assessing the therapeutic and toxicological effects of cesium chloride following administration to nude mice bearing PC-3 or LNCaP prostate cancer xenografts. Cancer Chemother Pharmacol. 2007;60(6):821-829.

7. Richardson MA, Sanders T, Palmer JL, Greisinger A, Singletary SE. Complementary/alternative medicine use in a comprehensive cancer center and the implications for oncology. J Clin Oncol. 2000;18(13):2505-2514.

8. Lyon AW, Mayhew WJ. Cesium toxicity: a case of selftreatment by alternate therapy gone awry. Ther Drug Monit. 2003;25(1):114-116.

9. Jones DL, Petrie JP, Li HG. Spontaneous, electrically, and cesium chloride induced arrhythmia and afterdepolarizations in the rapidly paced dog heart. Pacing Clin Electrophysiol. 2001;24(4 Pt 1):474-485. 